\title{
Le traitement du long terme et de la prospective dans les zones ateliers (suite) \\ Les rapports entre chercheurs et acteurs
}

\section{Séminaire du Programme "Environnement, Vie et Sociétés " du CNRS et de Natures Sciences Sociétés \\ (Meudon, 29 et 30 mars 2001). \\ Suite du compte rendu}

\section{BERNARD HUBERT}

La prise en considération du long terme est par définition une des dimensions majeures des recherches à mener dans le cadre des "zones ateliers". Cette introduction du temps long dans les démarches de recherche pose en particulier le problème du statut à accorder aux interventions des "acteurs " dans les processus qui sont l'objet de la recherche, à savoir les processus qui commandent les évolutions des ressources et des milieux naturels. Elles en sont en effet une composante à la fois essentielle et évolutive. Elles ont en outre leurs propres pas de temps et, de plus, leurs incidences sur l'" environnement "sont susceptibles de se faire sentir sur des pas de temps allant du court au long (voire au très long) terme.

Quand on s'intéresse aux évolutions passées, ces interventions humaines peuvent être tout simplement considérées comme des variables de ce qui s'observe et être analysées en tant que telles. Le problème est de passer du rétrospectif au prospectif, car la question posée au sein des "zones ateliers » est bien de tirer des analyses du passé des projections sur l'avenir. Et ceci, tout particulièrement, précisément, sur le long terme et en vue de l'action. C'est alors que se pose le problème de la conception d'une démarche de recherche capable de prendre en charge la nécessité d'articuler une problématique de l'analyse et une problématique de l'action.

C'est cette question que l'auteur du texte qui suit avait été invité à traiter oralement lors du séminaire. Ce texte est une version revue et corrigée de ce qu'il avait exposé. II fait suite aux textes déjà publiés par NSS à propos de ce séminaire (cf. NSS 3-2001, 4-2001, 1-2002, 3-2002).

\begin{abstract}
Dans le cadre de ces réflexions sur les « zones ateliers », il a été jugé souhaitable de traiter de la question des « rapports entre chercheurs et acteurs n. Cette question mérite effectivement d'être soulevée compte tenu de l'inévitable implication des chercheurs engagés avec d'autres partenaires dans des dispositifs d'action collective, car ce sont, en général, les partenaires qui sont en situation d'action et rarement les chercheurs seuls... Ces situations exigent qu'une attention particulière soit portée à la traduction des problèmes posés par ces partenaires en questions de recherche,
\end{abstract}

à la construction des objets de recherche permettant de les traiter, aux types de connaissances, qui vont être produites, tant du point de vue de leur valeur scientifique que de celui de leur utilité pour nos partenaires. Cette question se pose avec d'autant d'acuité que les problèmes d'environnement se construisent, pour la plupart, sur la base de connaissances scientifiques (changement climatique, trou dans la couche d'ozone, polluants dans les eaux de boisson, érosion de la biodiversité, etc.). Ainsi se constituent des dispositifs divers (Mormont, 1996) au sein desquels ces

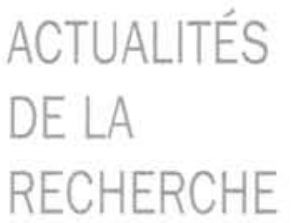

$\overline{\text { BERNARD HUBERT }}$

Écologue

Inra, département SAD,

147, rue de l'Université, 75338 Paris cedex 07 , France

hubert@avignon.inra.fr 
connaissances sont convoquées, à la fois pour constater le problème et pour lui trouver des solutions !

En fait, bien peu d'éléments sont disponibles en termes de cadrages théoriques, de propositions méthodologiques, de réelle prise en compte de ces démarches dans les systèmes d'évaluation, en particulier en ce qui concerne les chercheurs naturalistes ou biotechniques, pour les aider à répondre à ces sollicitations dans la conduite concrète de leurs travaux ; d'où une certaine frilosité des chercheurs à adopter de telles postures, voire un amateurisme dangereux professionnellement et institutionnellement pour certains de ceux qui s'y lancent naïvement. Ces nouvelles approches reposent sur des épistémologies constructivistes qui ne sont pas moins rigoureuses ni moins exigeantes que les épistémologies positivistes habituelles; elles ont leurs canons propres et parfaitement identifiables, mais qui ne sont pas appréciables à l'aune des postures classiques d'administration expérimentale de la preuve. On entre dans une épistémologie à trois options : le vrai, le faux et le possible. Quand on travaille avec des acteurs humains, ce qui devait se produire - ce qui est possible - ne se produit pas forcément, parce que justement les acteurs peuvent apprendre à orienter les événements vers d'autres issues du fait qu'ils ont amélioré leur état de connaissance de la situation grâce à leur interaction avec des chercheurs! Ils ont appris... C'est bien d'ailleurs là un des enjeux importants des exercices de prospective, mais c'est également un des points essentiels à prendre en considération au moment de la conception de nouveaux programmes de recherche.

Cet exposé vise à montrer que ces apparentes difficultés peuvent être transformées en de véritables atouts, que la recherche en partenariat, à condition qu'elle soit conduite avec rigueur et méthode, peut permettre d'aborder des objets complexes qui ont sens à la fois pour les chercheurs et pour leurs partenaires. Il sera constitué de cinq parties : la question de l'identification des partenaires, la clarification des postures de travail des chercheurs, la nécessité de reconnaître et d'assumer la diversité des points de vue, l'incidence de ces points de vue sur les modes d'action et, enfin, la construction des objets sociotechniques par l'engagement dans l'action collective.

\section{Partenaires et destinataires}

Dans les opérations de recherche "sur problème " conduites en partenariat, les premiers partenaires institutionnels des chercheurs sont ceux qui les ont interpellés en leur posant le problème à traiter. Mais bien souvent ces premiers partenaires se posent en porteparole de groupes sociaux ou professionnels. On ne sait pas toujours à ce stade si le problème est effectivement posé par ces groupes ou seulement porté par ceux qui s'en font les porte parole. La question qui se pose alors au chercheur est celle de la mise en place d'un dispositif de travail approprié à cette situation,

1 Classiquement évaluées par les institutions de recherche... tivement des problèmes que se posent ceux qui sont censés bénéficier du résultat des recherches. C'est cette question que je propose de contribuer à éclairer par les réflexions qui suivent et qui s'inspirent d'une analyse collective d'une opération de recherche en partenariat réalisée au Pays Basque, il y a quelques années (Hubert et al., à paraître).

Les dispositifs de recherche dont il est question ici visent donc à satisfaire différents partenaires, qui n'en attendent pas, en fait, les mêmes résultats, voire les mêmes retombées. Pour clarifier cette situation il est utile de distinguer plusieurs catégories de destinataires parmi ces partenaires : les commanditaires, les bénéficiaires et les institutions et opérateurs de terrain (figure 1).

Ceux qui sont habituellement désignés comme des " commanditaires " de la recherche sont bien souvent des institutions (ministères, Union européenne, collectivités territoriales, etc.) qui disposent de moyens financiers et d'une légitimité pour mobiliser des équipes de recherche. Les relations entre ces deux parties - commanditaires et chercheurs - sont les plus souvent formalisées par des conventions, des contrats, élaborés autour de termes de référence, plus ou moins précis, issus de négociations, d'aller retour à partir d'un appel d'offre ou de discussions, formelles ou non. Cette phase de négociation est essentielle, elle peut durer plusieurs mois et comprendre un moment particulier dédié à une analyse de situation réalisée sur le terrain, par un petit groupe de chercheurs de différentes disciplines se donnant les moyens de rencontrer un certain nombre d'autres acteurs locaux, individuels ou institutionnels, que les seuls commanditaires. Les documents contractuels qui en résultent, incluent généralement une clause de remise d'un rapport écrit au commanditaire ; ce rapport peut se limiter à des résultats de recherches au sens classique, mais il peut également inclure la référence à d'autres formes de produits (méthodes, documents de transfert et de vulgarisation, documents audiovisuels, compte rendu de réunions dites de restitution, etc.). Ce rapport clôt le contrat. En fait, le jeu ne se constitue-t-il pas souvent plutôt à trois? Les contrats sont effectivement signés par des représentants officiels des établissements de recherche (ainsi, par exemple, la procédure de « zones ateliers s est bien portée par le CNRS en tant qu'organisme public) : pour les chercheurs, satisfaire le commanditaire, c'est donc aussi donner satisfaction à son institution et on sait bien que cela se traduit par la reconnaissance des équipes et l'affectation des moyens. Mais n'est-ce finalement pas là une figure assez commune : les équipes de recherche sont évaluées d'abord par des procédures institutionnelles, sanctionnées par le maintien et le développement des équipes ainsi que par l'attribution de moyens de travail. N'y a-t-il pas en fait toujours un tel jeu à trois? Les établissements de recherche sont bien d'une certaine manière des commanditaires, qui assurent au moins les salaires des chercheurs et, en conséquence, leur demandent des comptes sur leurs activités.

L'évaluation des démarches de recherche sur problème conduites en partenariat soulève alors des difficultés, pour tout ce qui concerne leurs productions autres qu'académiques ${ }^{1}$, celles qui débouchent sur des 
propositions opérationnelles pour les partenaires, et dont le domaine de validité peut se révéler fort restreint. Les établissements de recherche ont du mal à évaluer ce type de travaux et surtout à les comparer aux démarches de recherche plus classiques, évaluées sur la base du nombre de publications scientifiques dans des revues à comité de lecture. Des propositions pour d'autres formes d'évaluation de ce type de recherches, de manière à identifier leur pouvoir génératif, ont été formulées par ailleurs (Hubert et Bonnemaire, 2000) ; le propos de cet article n'est pas de développer ces réflexions, même si cette question de leur évaluation n'est pas séparable de l'incitation qui est faite, de manière récurrente, à engager les scientifiques dans la conduite de telles recherches.

A priori, une deuxième catégorie de partenaires est celle qui est constituée par les « bénéficiaires ", au nom desquels le programme a été élaboré. Derrière cette catégorie se retrouvent des ensembles d'agents, souvent hétérogènes, ne s'identifiant pas systématiquement en tant que tels, ayant leurs propres modes d'organisation voire de représentation... II peut s'agir d'agriculteurs, de consommateurs (de produits divers ou tout simplement d'eau potable), de touristes et autres amateurs de paysages, d'associations naturalistes... voire même des générations futures ! Alors, comment les contacter, les identifier correctement et comment s'assurer que leurs problèmes sont bien ceux qui nous ont été exposés? Certes, une analyse de situation préalable peut donner quelques garanties sur la pertinence des termes de référence, mais elle ne permet pas toujours d'évaluer avec rigueur la complexité de la situation locale : conflits entre groupes, formes des relations sociales entre eux, poids et fondements des cultures locales, historicité des résistances, jeu des institutions, dynamiques locales, interactions avec les dynamiques englobantes, etc. Il nes'agit certes pas d'étudier tout cela, mais de s'assurer de ne pas intervenir à contre sens et que les propositions qui résulteront des recherches bénéficieront bien à ceux à qui elles sont formellement destinées : il s'agit bien de prendre en compte ces "bénéficiaires " aussi bien individuellement que dans leurs diverses formes de relations et d'organisation. Les écueils portent alors sur le risque de ne les mobiliser que pour améliorer le diagnostic initial de la recherche et construire sa problématique, ou seulement pour collecter des données " réelles " sur le terrain. Comment coopérer à ces différentes phases et quels dispositifs mettre en place pour ce faire entre des chercheurs, dont le métier est de produire des connaissances nouvelles à partir de procédures formelles, et des acteurs divers, qui manipulent d'autres formes de connaissance dans l'action ${ }^{2}$ ?

Enfin, une troisième catégorie de partenaires doit bien souvent être également prise en compte : celle des organismes et institutions opérateurs sur le terrain. Ces institutions sont le plus souvent en position intermédiaire entre des bailleurs de fonds dont elles dépendent (État, Union européenne, collectivités territoriales, organismes consulaires, etc.) - qui sont également les " commanditaires " - et les professionnels ou les usagers auprès desquels elles interviennent comme

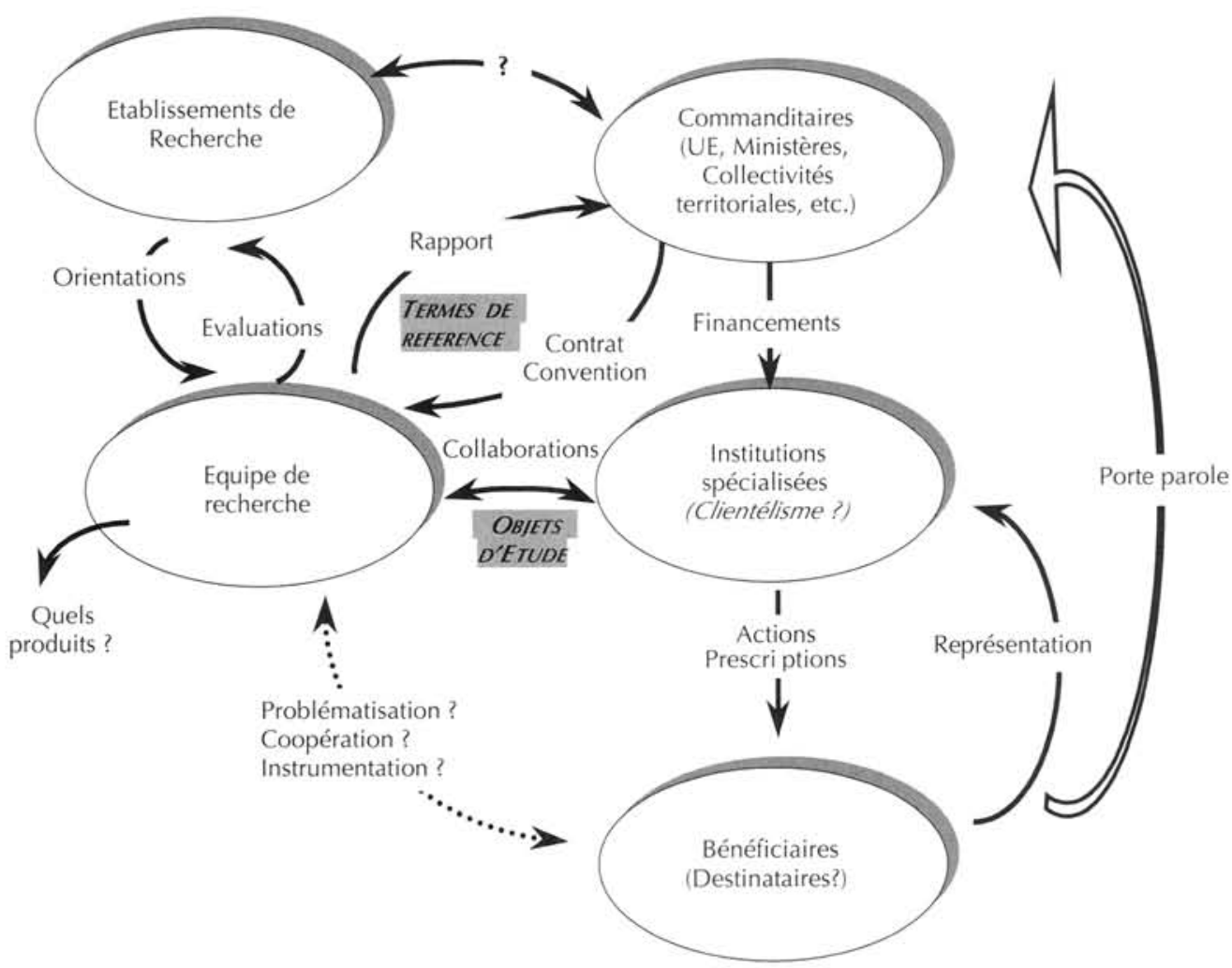

Figure 1. Réseau chercheurs/partenaires : quels destinataires? 
des prescripteurs. Elles deviennent incontournables du fait de cette position comme de leurs compétences spécifiques dans leur domaine d'action, voire de leur connaissance du contexte local. En outre, une connivence s'installe rapidement avec les agents de ces institutions qui sortent des mêmes universités ou grandes écoles que beaucoup de chercheurs, qui ont des positions sociales voisines et le même rapport à la légitimation scientifique du discours technique; ce qui n'est pas le cas de la plupart des autres acteurs. II peut alors se révéler particulièrement facile et pertinent d'en faire les destinataires des résultats de recherche, puisqu'ils sont localement en prise directe avec l'action du fait de leur position de prescripteurs, de leur pérennité (alors que les chercheurs vont quitter le site à plus ou moins brève échéance) et de la plus grande facilité à leur transmettre des outils directement utilisables, car accessibles culturellement (ils manipulent, comme les chercheurs, les abstractions, l'écrit, les cartes, les modèles, les instruments informatiques, etc.). On peut classer dans cette catégorie les gestionnaires de parcs, les agences, les services administratifs (DDAF, DDE, ONC, ONF), les Instituts techniques, les services des Chambres d'agriculture, des collectivités territoriales, certaines associations de protection de la nature, des ONG, etc.

Le type de catégorisation auquel renvoient ces considérations est voisin de celui proposé par Checkland (1993) pour la conduite d'une démarche systémique appliquée à des situations diverses (le plus souvent hors du monde agronomique et rural) et qu'il appelle " root definition "; ce qui est en cause selon lui, c'est l'identification des structures de base d'un système - si tant est qu'on puisse l'exprimer ainsi - qu'il identifie autour de six points principaux repérés par les six lettres du sigle CATWOE :

- C pour "Customers " désigne ceux qui sont directement touchés par les transformations du système, bénéficiaires ou victimes ;

- A pour «Actors» représente les agents qui agissent directement sur les transformations du système :

- T pour le processus de "Transformation " qui donne sens au système étudié ;

- W pour la "vision du monde " ou "vision des choses ", "Weltanschauung ", sur le système ;

- O pour "Owners " désigne en quelque sorte les " propriétaires " du système, ceux qui ont le pouvoir de le développer, le réduire, ou vouloir le transformer et faire appel à des tiers pour les aider dans leurs intentions ;

- E pour l' "Environment " du système et les contraintes engendrées sur celui-ci.

Les trois catégories d'agents distingués dans cette approche (Owners, Actors, Customers) ne sont pas bien loin de la distinction entre "Commanditaires", "Organismes opérateurs " et "Bénéficiaires ". Toutefois, $P$. Checkland prend bien la peine de préciser que plusieurs « visions du monde » (Weltanschauung) peuvent être développées à propos du même système. Et ces visions du monde peuvent varier au sein même des trois catégories d'agents et d'institutions, O, A et C. Mais, justement, ces catégories ne sont pas figées, elles peuvent évoluer selon la vison du monde considérée ainsi qu'en fonction de la dynamique de la situation.
Certaines actions entreprises peuvent justement viser à transformer en * Actors * des agents dominés en situation de "Customers", ou plus souvent à rendre les "Actors " habituels plus pertinents dans leurs interventions. C'est bien dans la dynamique de l'action collective impliquant des chercheurs et ces différents partenaires que de telles transformations se réalisent : le concept flou "d'aide à la décision " prend alors sens dans des interactions diverses, qui ne reposent pas de manière systématique sur des instruments ad hoc formalisés. D'autant que chacun de ces partenaires est engagé dans des " régimes d'action " différents, c'està-dire qu'ils mobilisent et articulent des ensembles distincts de ressources (Dodier, 1993). II est donc essentiel de veiller à la façon dont nous-mêmes, chercheurs, ainsi que les connaissances que nous produisons, sommes alors mobilisés en tant que ressources. On mesure donc tout l'intérêt qu'il peut y avoir à ne pas mélanger ces différentes catégories sous le vocable commode de " partenaires " !

L'équipe de recherche est donc amenée à constituer des dispositifs d'intervention, plus ou moins complexes pour gérer et institutionnaliser ses relations avec ces différentes catégories de partenaires. C'est ainsi, par exemple, qu'au Pays basque, nous avons constitué (1) un " comité de pilotage " avec les représentants des institutions, (2) des "groupes de travail " avec les agents des opérateurs intermédiaires et (3) des * groupes locaux " avec les agriculteurs (Hubert et al., à paraître) ; de même, dans les Cévennes, nous avons travaillé avec une "cellule technique», un " groupe restreint » et un " groupe élargi » (Couix et Hubert, 2000; Couix et Hubert, à paraître).

Mais le travail des chercheurs ne va pas être le même à ces différents niveaux du dispositif; il va varier selon leur discipline, selon le type de question à traiter, donc selon les objets de recherche identifiés, les méthodes pour les aborder, les techniques de collecte des données, etc.

\section{Clarifier les postures de travail}

A. Hatchuel (2000a) distingue dans le travail de recherche trois " modèles ", qui correspondent chacun à des conditions différentes d'exercice des activités professionnelles et d'implication avec les différentes catégories de partenaires:

- Le "modèle de laboratoire ": c'est le modèle le plus classique du type de recherches conduites en agronomie, en physique, en biologie. II repose sur la considération que le monde est vaste et complexe et qu'il faut en isoler une partie close sur elle-même afin de pouvoir l'étudier : le chercheur va donc en extraire un morceau et le séparer du reste du monde. C'est la culture de l'expérimentation. On reconstruit ainsi une situation expérimentale sur laquelle il est possible d'agir puisqu'on procède alors à une "manipulation " : on agit sur des facteurs dits * de contrôle " afin de mettre en évidence les conséquences de l'action de ces facteurs sur le processus étudié. On a défini préalablement une hypothèse sur le rôle de ces facteurs de contrôle, un protocole pour éprouver ces hypothèses 
ainsi que les critères de performance de l'expérimentation qui permettent d'en évaluer les résultats. Un protocole est ainsi conçu afin de manipuler tel ou tel facteur et de mesurer son effet à la sortie, de façon à confirmer ou invalider les hypothèses qu'on s'était données sur le rôle de ce facteur. Dans cette posture, les connaissances sont produites par des chercheurs, $c^{\prime}$ est-à-dire les professionnels de la production de connaissance scientifique, ceux qui maîtrisent l'expérimentation. Les résultats sont publiés et diffusés afin qu'ils puissent servir à leurs utilisateurs potentiels.

- Le " modèle de terrain »: il est venu de l'écologie et des sciences économiques et sociales. II reconnaît que le monde ne peut pas être clos, qu'il est complexe et qu'il repose sur un grand nombre d'interactions. Le chercheur va aller l'étudier tel qu'il est, dans la nature ou dans la société, en utilisant des objets, ou artefacts, pour en rendre compte. C'est le cas, par exemple de l'écosystème, conçu pour étudier les interactions et les flux dans les systèmes naturels ; une partie des modèles économiques sont également conçus sur ces bases, pour représenter d'une manière abstraite - non pas physiquement ${ }^{3}$ - le fonctionnement du monde. Ainsi on ne touche pas le monde réel, mais on se construit un modèle à partir du point de vue sur le monde dont on veut rendre compte. On procède alors à des observations, et non pas à des expérimentations. Les critères de performances ont été identifiés ex ante et ils ont été pris en compte dans la conception des protocoles d'observation et des modèles utilisés pour représenter les processus étudiés et interpréter les résultats. Les connaissances produites sont publiées et diffusées afin d'être appliquées en dehors du monde de la recherche.

- Le « modèle de la recherche-intervention " qui vient des sciences de gestion, des démarches ethnométhodologiques ou dites compréhensives en sociologie : il assume que le chercheur n'est pas hors du monde et qu'il est aussi acteur du monde qu'il étudie. Produire de la connaissance sur le monde est aussi une façon d'être acteur du monde. Plutôt que de se contenter de reconstituer une partie du monde dans son laboratoire ou de construire des modèles pour le représenter selon certains points de vue, le chercheur se met lui-même en situation d'interaction, en s'engageant dans l'action collective avec les partenaires qui ont fait appel à lui. Les critères de performance, permettant d'évaluer ce qui se passe dans ce type de recherche et ce qui en émerge de nouveau, ne sont pas forcément déterminés à l'avance. Souvent, on ne sait pas ce qui va sortir concrètement de ce type de recherche, même si on connaît les problèmes qui ont conduit à la coopération entre des chercheurs et des partenaires sociaux. Ces critères de performance doivent néanmoins être explicités ex post de façon à démontrer que quelque chose de réellement nouveau a été apporté pour la compréhension d'une situation et la résolution d'un problème. Dans cette posture, les connaissances sont produites dans l'action, en interaction entre le chercheur et ses partenaires. Il y a une méthode, mais pas forcément de résultats pré-programmés, et les critères qui vont permettre de dire comment il faut s'y prendre ne pourront être définis que quand le travail commun aura été avancé ; avant, on ne sait pas. Un tel processus repose sur des apprentissages croisés entre tous les participants et ce genre de méthode a des chances de s'enrichir et de se préciser à chaque étape de sa mise en œuvre et à chaque nouvelle application qui en est faite.

Ainsi, il y a une relation directe entre les types de posture adoptée par les chercheurs, les modalités de production de connaissances et les connaissances ainsi produites. En fonction des postures de travail qu'on a choisies, et qui sont liées à la discipline de chacun, à son histoire et à sa culture, on ne va pas produire les même types de connaissance. Cette clarification est importante, car se tromper, croire se trouver dans un autre contexte paradigmatique que celui dans lequel on est effectivement, génère des controverses qui mènent à des impasses (car on ne peut plus se comprendre) ou aboutit à des solutions inacceptables (car à contre-emploi).

La diversité des partenaires se combine ainsi à une diversité des postures des chercheurs, mais cette apparente difficulté permet en réalité de constituer les dispositifs opérationnels, c'est-à-dire intégrant connaissances et actions. La perspective de construction d'objets sociotechniques devient alors organisatrice de ces diversités afin d'en faire émerger de potentielles synergies.

\section{Assumer la diversité des points de vue...}

Ainsi, on agit selon ce qu'on a compris du monde et selon les concepts qu'on a pour se le représenter. Dans une telle perspective, il est indispensable que les chercheurs eux-mêmes aient une idée claire du statut qu'ils donnent à leur démarche de recherche, du type de connaissances qu'ils produisent, du mode d'intervention qu'ils mettent en œuvre, du type de partenariat qu'ils engagent. La notion de " point de vue " est utilisée ici dans le sens employé par Legay (1993, 1997) et Pavé (1994) pour désigner des démarches de recherche sur des systèmes complexes privilégiant une entrée spécifique choisie pour sa pertinence compte tenu d'une finalité donnée. Il s'agit là d'un "point de vue "scientifique, c'est-à-dire construit, choisi, à la différence de ce que sont les points de vue de la vie quotidienne, non construits par un acte délibéré, mais définis, selon la définition du sémiologue L.J. Priéto (1975), par les activités des sujets et leurs positions par rapport à une réalité ; plusieurs points de vue sont ainsi bien entendu possibles, de même que le sont différentes "Weltanschauung " évoquées par Checkland (op. cit.). Dans une réflexion sur ces formes de coopérations entre des chercheurs et des praticiens, Darré (1997) parle d'une "double distance ": d'une part, entre les " points de vue " des uns et des autres sur la réalité et, d'autre part, entre la nature et le niveau d'élaboration des problèmes que les uns et les autres formulent.

Pour illustrer cette différenciation des points de vue, je prendrai pour exemple les questions que posent les concepts du développement durable, appliqués à la
${ }^{3}$ Alors que, quand on fait une expérimentation, on découpe une partie du monde qu'on apporte ou reconstitue au laboratoire ou sur des parcelles expérimentales. 


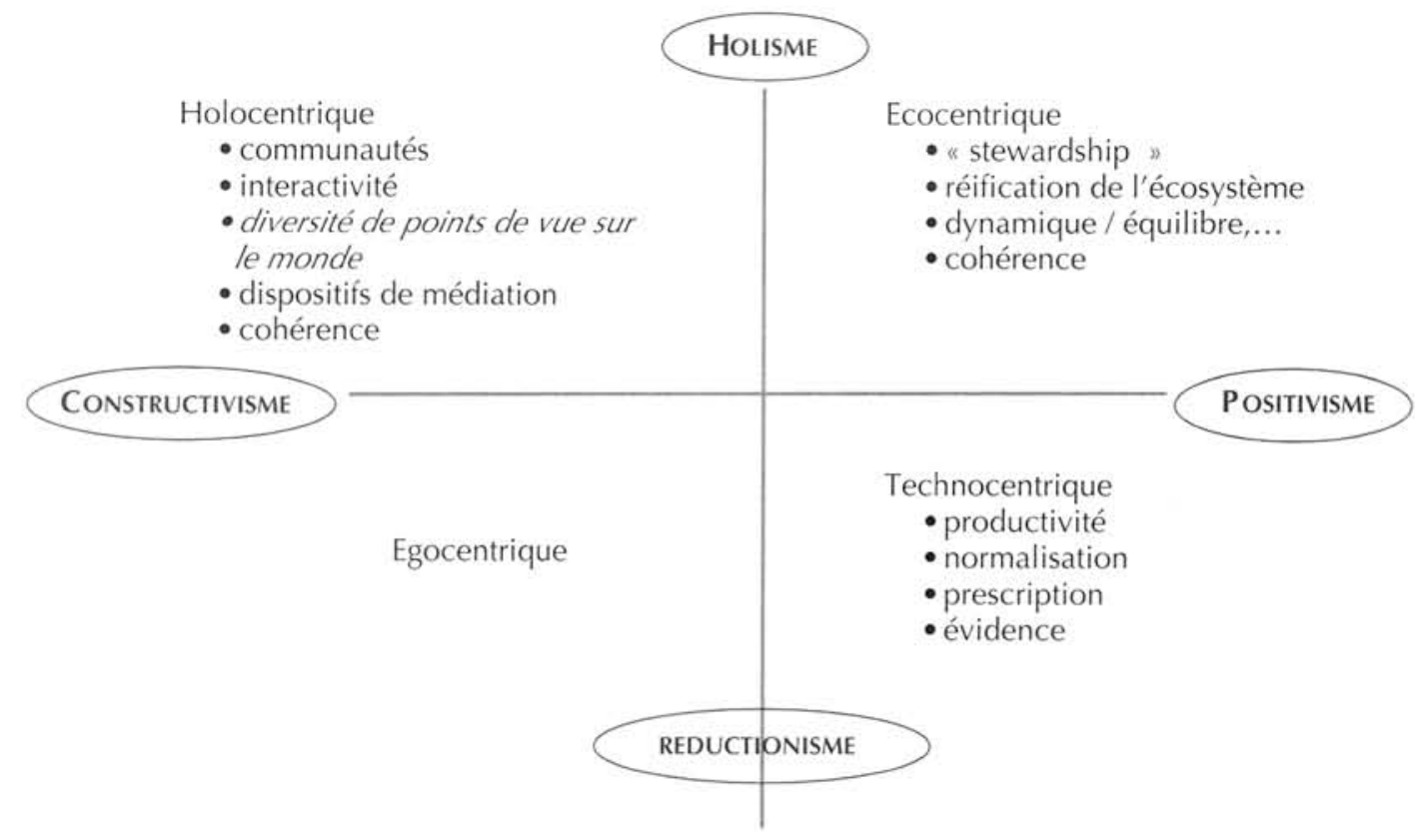

Figure 2. Les quatre points de vue sur la gestion des ressources naturelles renouvelables selon R. Bawden (1997).

notion de système de production en agriculture. Elles portent sur de multiples facettes de leur conception même : leur configuration autant que leur pilotage, la diversification des produits, les accords avec les autres opérateurs, la sécurisation des conditions de production, etc. Ce ne sont pas là autant d'éléments qu'il faudrait ajouter les uns aux autres pour enfin atteindre une compréhension exhaustive de leur fonctionnement, car cette vision totale est inaccessible. Mais il est indispensable d'apprendre à regarder ces aspects de leur fonctionnement autrement, en associant des points de vue différents et complémentaires, ainsi que le recommande Richard Bawden (1997); celui-ci invite à dépasser une vision strictement technologique et anthropo-centrée, habituelle en agronomie, tout en évitant de s'enfermer dans une analyse qui ne serait par contre qu'éco-centrée, ne privilégiant qu'une stricte approche naturaliste. Pour cet auteur, il s'agit de clarifier ces oppositions, afin d'éviter les confusions paralysantes et de replacer dans leur contexte sociétal les valeurs qui accordent leur durabilité aux faits. II est, en effet, important dans un premier temps de bien comprendre que derrière les notions de développement durable ou de gestion durable des ressources, selon les points de vue mobilisés, les objets peuvent ne pas du tout être les mêmes, bien que parfois dénommés de la même façon : on peut alors n'y rien comprendre et ne pas pouvoir passer à l'action. Selon que I'on considère l'eau comme ressource-stock ou comme élément fonctionnel d'un système, on n'entend pas la même chose et l'on ne peut s'accorder ni sur le diagnostic, ni sur les actions à entreprendre. Comment alors changer de point de vue ou créer des termes de passage entre points de vue ? Il convient donc de disposer d'une grille d'analyse pour se re- trouver dans cette diversité des perceptions de ce qui paraît important, et des façons dont les acteurs vont se situer pour agir.

Pour construire cette grille, on peut partir de celle proposée par Richard Bawden ${ }^{4}$, qui est constituée de quatre cadrans délimités par deux axes orthogonaux : un axe vertical qui oppose les visions réductionnistes du monde, vers le bas, et les visions holistes vers le haut, et un axe horizontal, qui distingue une vision objectiviste (ou positiviste), à droite, d'une vision constructiviste, à gauche. L'un de ces cadrans, celui situé en bas à gauche du tableau, n'est pas pertinent en termes de développement durable. R. Bawden voit respectivement dans les autres: un cadran " technocentré " (en bas à droite), un cadran "éco-centré " (en haut à droite), et un cadran " holistique " (en haut à gauche) (figure 2).

Le cadran " techno-centré ", comme son nom l'indique, est centré sur la technique comme voie de mise en valeur de la nature, il est caractérisé par la notion de productivité. C'est le paradigme qui a dominé la pensée agronomique depuis sa création, ou du moins, une partie dominante de cette pensée. C'est celui de la normalisation au sens de la production de standards et de références, ainsi que de la prescription comme mode de transmission des savoirs. C'est sur cette pensée qu'a été construit l'essor de l'agriculture française pendant 40 ans. Elle est fondée sur l'objectivité : la réalité, c'est la vérité. Elle repose sur des connaissances " objectives ".

Le cadran " éco-centré » est celui d'une perception qui est davantage celle de l'écologie, au sens scientifique du terme. On n'y recherche pas directement la productivité, mais plutôt comment on gère les fonctionnements naturels, comment on préserve l'intégrité 
des processus biologiques et des cycles géochimiques sur lesquels reposent ces fonctionnements. La nature n'est plus seulement au service de l'homme, il faut également que l'homme en respecte certains fonctionnements et dynamiques. La notion d'écosystème est ainsi un concept théorique produit par les écologues pour se représenter le monde et son fonctionnement, ses cycles, ses équilibres et ses dynamiques; mais l'écosystème n'a pas de dimension spatiale. On peut très vite alors être tenté de réifier les écosystèmes et de se dire, par exemple, qu'une mare - ce qui est facile, car bien délimité - ou une prairie - ce qui est plus difficile, car en continuité avec des chemins, des bordures, des haies - sont des écosystèmes. Certes, on peut rendre compte du fonctionnement d'une mare par un modèle éco-systémique, mais une mare n'est pas un écosystème en soi. On va trouver aussi des concepts de dynamique (de population, par exemple), d'équilibre. Ce n'est plus l'objectivité des choses - comme dans le cadran précédent - qui va compter mais leur cohérence, c'est-à-dire la pertinence des interactions identifiées qui lient les éléments. L'apport de la science écologique consiste ainsi à étudier ces interactions et les fonctionnements qui en dépendent; elle analyse les cycles, leurs dynamiques, leurs rythmes, leurs entrées et leurs sorties. C'est bien une question de cohérence, de bilan et non plus d'une objectivation absolue des choses du monde. II n'y a plus de vérité en soi, mais des connaissances plus ou moins abouties, et donc des incertitudes, sur la complexité du monde...

Le cadran " holistique " est celui des approches privilégiant les interactions sociales et les "solidarités", celles-ci comprenant aussi bien les alliances que les oppositions ; on va rechercher alors autant ce qui intéresse les gens, ce qui les relie au monde tel qu'ils se le représentent et, donc, le connaissent. II ne s'agit plus seulement de prendre en compte l'existence de fonctionnements naturels, mais également les activités, les pratiques, les intentions et les projets des groupes humains qui tirent leurs ressources de ces fonctionnements : il n'est plus de systèmes naturels en soi, mais des systèmes de connaissance et d'action sur le monde. Interviennent alors essentiellement les interactions entre les individus et entre les groupes sociaux, leur contenu, leur puissance, leur force et leur nombre ainsi que les transformations induites par ces interactions au cours du temps. La diversité des points de vue sur le monde devient incontournable, puisqu'on en privilégie un là, ou un autre ailleurs : certains, même, ne voient pas la nature comme aussi distincte des humains que ne l'a établi la pensée occidentale. Il s'agit alors de reconnaître ces différences et le fait qu'on a besoin de connaissances et de concepts dans ces différents systèmes de pensée (ce qui inclue les autres cadrans) pour agir sur le monde ; c'est le rôle des dispositifs de médiation, et des formes de représentation de ces différents systèmes de pensée, que de permettre de tels compromis. On est bien là également dans un monde de la cohérence, pas forcément dans un monde de l'objectivité, à partir du moment où on reconnaît qu'il y a une diversité de points de vue, qu'ils ont chacun leur pertinence en regard de qui les porte et les exprime. Il y a alors plusieurs vérités...
En circulant dans cette grille - du bas à droite vers le haut à gauche - on gagne en réflexivité et en appréciation de la durée des processus temporels en cause. La réflexivité s'accompagne ainsi d'une plus grande prise en compte du long terme.

\section{...et leurs conséquences sur les modalités de l'action}

Mais ce sont également les principes et les modalités du rapport à l'action qui ne sont pas les mêmes d'un cadran à l'autre.

En termes de procédure d'action (figure 3), quand on agit dans le cadran * techno-centré » exclusivement, on est dans des procédures qui reposent sur des formes d'organisation hiérarchique, de la connaissance normalisée, ce qu'on a appelé des références, produite, dans le monde de l'agriculture, par la recherche publique et des instituts techniques spécialisés. De plus, l'ensemble a été constitué comme un domaine protégé, différencié des autres : on a ainsi fait fonctionner le domaine agricole et agro-alimentaire comme un isolat dans la société française, avec des output spécifiques, les biens nourriciers livrés au reste de la société, dont les autres préoccupations étaient gérées autrement, par d'autres procédures au sein d'autres domaines également spécialisés. C'était un "domaine réservé ", avec ses professionnels et leurs organisations, son dispositif de formation propre, ses corps de l'État, son ministère spécialisé, un établissement de recherche et des instituts techniques, un système d'accompagnement et d'appui technique, etc. ${ }^{5}$. Ce modèle fonctionne selon une organisation planifiée (au sens de Dodier, 1997) selon laquelle « la concrétisation et le fonctionnement des objets obéit à des lois dont les concepteurs, en tant que scientifiques, ont une connaissance qui leur assure la maîtrise des réseaux, si tant est que les opérateurs s'alignent sur leurs plans ». Les procédures de fonctionnement des réseaux sont considérées comme stables, établies par les concepteurs détenteurs du savoir fonctionnel nécessaire. Ce qui a bien été le cas pendant 50 ans... et a abouti, par exemple, à la « modernisation " de l'agriculture française ou à la "révolution verte". Quand on travaille dans un tel cadre, les formes de réaction aux incertitudes ou aux transformations de l'environnement relèvent de ce qu'on peut appeler des * changements de premier ordre " : la solution à un problème y revient, en gros, à revoir les routines appliquées, à changer les règles ou à ré-ajuster les fameuses références. On peut, par exemple, convenir de revoir des normes réglementaires, à la hausse ou à la baisse, après avoir validé le constat que les normes actuelles ne sont pas applicables ou qu'elles sont peu pertinentes. On ne change ni les réseaux d'acteurs, ni les procédures qui les animent, ni les modes de production des connaissances.

Dans le cadran " éco-centré ", les procédures passent par des formes non-hiérarchisées d'organisations de type délibératif, comme des forums, ouvertes à des débats. Ces organisations délibératives reposent sur ce qu'on appelle des réseaux socio-techniques, au sein desquels des acteurs divers se retrouvent et débattent
${ }^{5}$ D'ailleurs ce modèle, assez spécifique à notre pays, ne s'est pas seulement appliqué en agriculture. 
ACTUALITÉS entre eux d'entités très hétérogènes comme leurs systèmes de valeurs, des normes, des objets, autour des systèmes écologiques et des processus mis en discussion (Deverre et al., 2000). Ce sont des cadres souples qui organisent l'action, les confrontations et les associations entre acteurs. Ces forums sont ouverts à des étrangers au domaine concerné et au système débattu : c'est l'argumentaire du problème en cause qui définit les droits d'accès à la délibération et les frontières, ce qui peut donner lieu à controverse. Les divers énoncés des uns et des autres sont ainsi l'objet de traductions au sein du collectif, permettant leur partage et leur socialisation : ces traductions permettent la mise en équivalence de ressources hétérogènes, d'intérêts a priori incommensurables et qui supposent des ajustements entre acteurs dans la définition même de ce qu'ils sont (Callon, 1989). C'est un processus dynamique, fait de rapports de force et de négociations pour détourner des intérêts et créer des alliances autour d'un problème, qui permet en outre aux acteurs d'effectuer des rapprochements avec d'autres situations, et ainsi de conduire l'action et d'établir un jugement sur son accomplissement (Akrich, 1993). Les adaptations, les transformations ou les solutions des problèmes relèvent alors de * changements de deuxième ordre » : on ne va pas uniquement déboucher sur des changements de standards ou de règles, mais on va éventuellement remettre en cause les principes et les normes qui sont à l'origine de ces règles. La solution au problème revient alors à le poser dans un espace reconstruit par la configuration élargie d'acteurs et dans de nouveaux termes issus de ces traductions successives : telle activité ou tel facteur mis en cause peuvent se révéler inopérants, il faut aller en interroger d'autres portant sur d'autres processus liés, mais qui n'étaient pas apparus au premier chef. Le réseau d'acteurs impliqués s'est ainsi transformé avec les objets en cause, permettant la recherche de solutions innovantes dans cette situation là. C'est sur de telles bases que se sont constitués les dispositifs de mise en place des premières mesures agri-environnementales comme les opérations dites " article 19 ", les "OGAF environnement " (Alphandery, 1996 ; Billaud, 1996 ; Mormont, 1996) ou que se constituent de nos jours les comités chargés de piloter localement les opérations Natura 2000.

Dans le troisième cadran, on est dans « l'action collective ", plus organisée que le forum. On passe à des formes d'organisation centrées sur les acteurs sociaux, on n'est plus dans une dominante écologique, qui s'imposerait au nom de la "nature ", mais dans l'analyse des rapports à la " nature » dépendants des connaissances disponibles, tacites comme explicites, des activités fondées sur ces connaissances et de leur aptitude réciproques à évoluer en situation d'interaction avec d'autres formes de connaissances et d'autres types d'activités. Ce qui compte alors, ce sont justement ces transformations en profondeur des connaissances - et pas seulement des énoncés comme dans le cas précédent - des uns et des autres du fait des apprentissages croisés permis par les dispositifs formels d'interaction. Ce sont alors plutôt des organisations dans lesquelles " on reconnaît comme inéluctable, constitutive des réseaux techniques, une certaine incertitude quant au fonctionnement des objets, et d'autre part que cette incertitude est gérée localement et publiquement, au fur et à mesure de la survenue des aléas, par des opérateurs considérés comme détenteurs de connaissances hétérogènes, non réductibles à un savoir central " (Dodier, 1997) ; les formes de connaissances, les concepts, les activités des différents participants y sont dûment identifiés et reconnus. Ce sont alors des

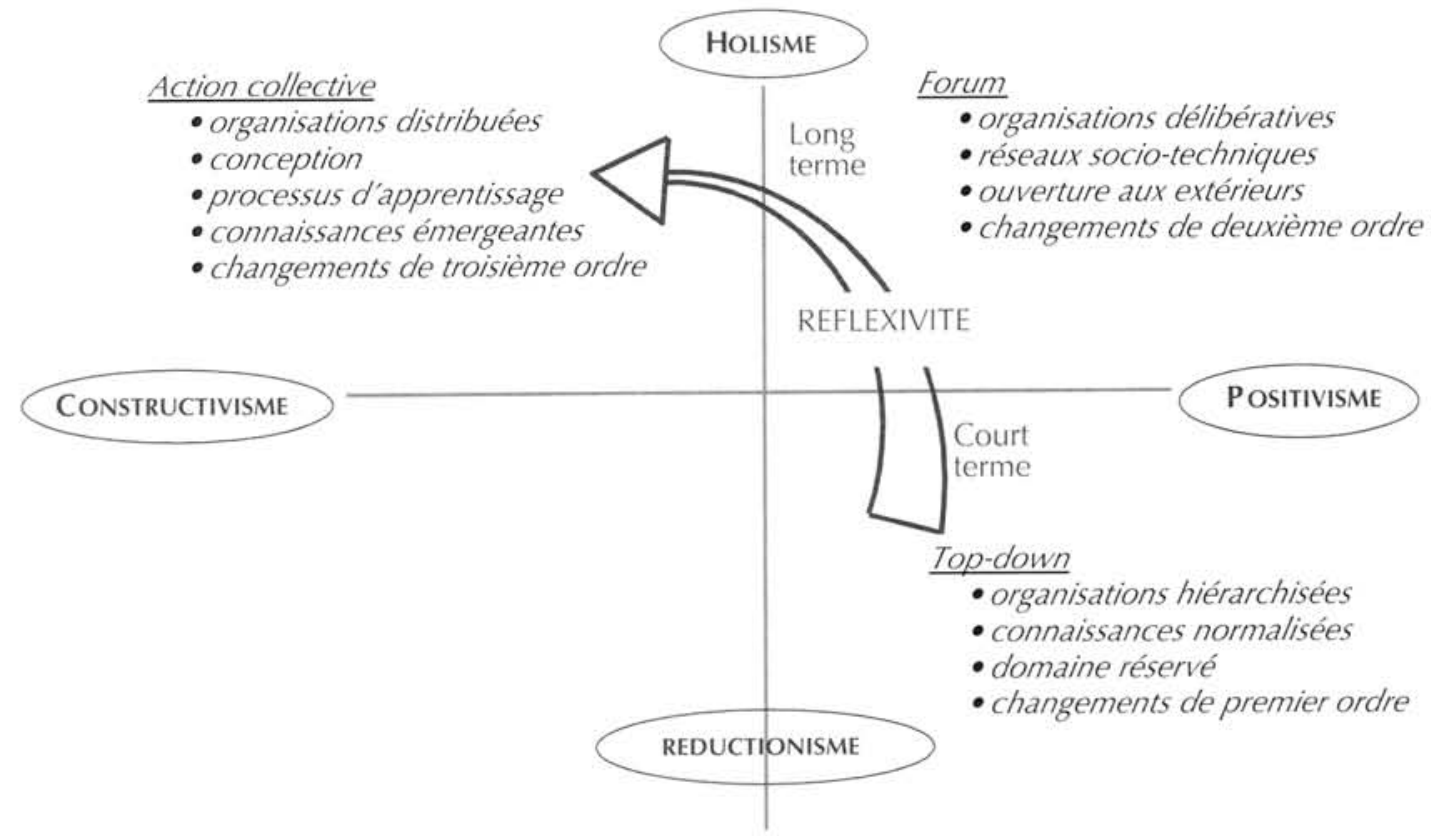

Figure 3. Formes et modalités d'action selon trois des quatre points de vue identifiés par R. Bawden (cf. figure 2). 
dispositifs formels qui permettent de répondre rapidement aux transformations incessantes par la confrontation des perspectives; I'exigence y est mise alors sur les capacités d'adaptation aux changements.

Il y a ainsi une forte orientation sur les enjeux de conception, c'est-à-dire de construction des cadres de raisonnement, qui sous-tendent la compréhension du monde, les processus d'apprentissage, la production de connaissances nouvelles émergentes, issues des interactions entre les participants. Quand des problèmes sont traités dans ce type de dispositif avec de tels principes et procédures, on n'en est plus à changer les routines et les règles, ni à remettre en cause les raisonnements qui sont à l'origine de ces routines, mais on en est souvent à remettre en cause les valeurs qui sont à l'origine de ces raisonnements et des principes qui les sous-tendent. On change alors le système de valeurs; c'est-à-dire que le problème tel qu'il était posé n'a peut-être plus lieu d'être, la situation, que certains posaient comme un problème est alors reconsidérée selon des points de vue différents. II n'y a peut-être plus problème ou bien celui-ci s'est déplacé vers d'autres questions : par exemple, la résolution de problèmes posés par des fonctionnement biophysiques peut passer par l'invention de nouvelles formes d'organisation des activités qui sont à l'origine de ces dysfonctionnements en imaginant et en réalisant des dispositifs inédits en charge de la gestion de nouveaux objets, construits de manière critique en référence aux processus examinés en premier lieu. Les réseaux d'acteurs se transforment en même temps que leurs formes d'organisation et l'état des connaissances partagées, aboutissant à des solutions inédites. Ces dispositifs reposent sur des fonctionnements plus complexes, avec plusieurs niveaux de conseils, comités ou groupes de travail ayant des tâches définies et différenciées (Deverre et al., 2000). Ainsi par exemple, dans la réflexion sur la reconstitution du Périmètre incendié des Cévennes gardoises, ont été constitués une « cellule technique ", un " groupe élargi " ainsi que des groupes de travail spécialisés et temporaires (Couix et Hubert, 2000 ; Couix et Hubert, à paraître) ; de même, la relance de I'agropastoralisme au Pays Basque intérieur a reposé sur un "comité de pilotage ", trois " groupes de travail "spécialisés, des "groupes locaux " impliquant les agriculteurs à l'échelle d'une commune, des sessions de formations des agents de développement, etc. (Hubert et al., à paraître). Le GIS "Alpes du Nord * fonctionne ainsi sur la base d'un comité de groupement, d'un conseil scientifique, d'ateliers thématiques spécialisés, de séminaires périodiques, etc. (Roybin et al., 2001).

Il ne s'agit pas de donner la primauté à ce point de vue " holistique » (d'autant que lui même reconnaît les autres), mais ce qui importe, c'est au moins de clarifier et de reconnaître ces différences de points de vue, et donc de formes de connaissances et de types d'enjeux qui leur sont liés. II s'agit de les clarifier afin de les mobiliser de manière complémentaire et de savoir où chacun se place, pour aller vers une vision du court terme au long terme et augmenter ainsi notre réflexivité sur ce qui est mis en œuvre, voire mis en question. Il en va de même pour les postures de recherche, qui s'inscrivent également dans cette catégorisation.
Quand on est dans le modèle de laboratoire, on produit essentiellement dans le cadran " techno-centré ". On produit des connaissances qui ne peuvent pas être facilement utilisées dans le cadran « éco-centré ». En effet, les critères et les modalités de production de connaissance du premier cadran ne sont pas directement compatibles avec l'approche écologique : prendre en compte les processus écologiques dans une démarche qui resterait strictement expérimentale, de type agronomique, passe par des réductions considérables - ne serait-ce que temporelles - qui risquent de passer à côté d'interactions mal identifiées mais pourtant cruciales... à moins de se contenter de mesurer l'effet de quelques facteurs ciblés, dont la contribution à un fonctionnement englobant aura été auparavant explicitée. II faut, même pour cela, changer de modalité de travail, et passer à des protocoles d'observation sur le terrain et à la modélisation. De même, vouloir aborder les questions du troisième cadran, " holistique ", ne peut se réaliser qu'en s'engageant, en tant que chercheur, dans l'action collective elle-même en situation, avec les autres partenaires concernés par un problème que cette situation leur pose. Callon (1998) qualifie cette approche de modèle, pragmatique de communication de la recherche avec les processus de décision ${ }^{6}:$ dans ce modèle les interactions entre systèmes de connaissance - des acteurs entre eux ainsi qu'avec les chercheurs - ouvrent de nouveaux champs d'action et de relations, qui permettent l'émergence de nouvelles " communautés " d'acteurs individuels et collectifs liés par des savoirs scientifiques et pratiques, des techniques, des normes, des préférences culturelles et des réalités naturelles... qui font alors évoluer en même temps les savoirs, les engagements et les réseaux d'échange.

Il ne s'agit pas de dire ici que telle posture est supérieure à telle autre ou plus scientifique que telle autre. Il s'agit d'assumer le fait que des objectifs différents ne peuvent pas être atteints à partir de n'importe quelle posture de recherche et que chacune de ces postures a des règles qui lui sont propres et qu'il faut respecter : c'est cela le critère de scientificité. En effet, ce ne sont pas les objets qui font la différence dans le travail de recherche, mais les démarches que les chercheurs mettent en œuvre et les méthodes qu'ils utilisent pour les étudier. Les problèmes complexes, comme ceux identifiés dans la plupart des zones ateliers, ne peuvent pas être traités dans les seuls cadrans " techno-centré » (le plus habituel dans la culture agronomique) ou * écocentré » (pour les écologues), le reste relevant alors d'un habillage « sociologique », voire «politique »... Il est alors nécessaire qu'à un moment donné du déroulement de la recherche, certains chercheurs, au moins, s'engagent dans des approches interactives avec les partenaires des projets. C'est cela qui permet l'identification et la construction des objets socio-techniques, qui seront ensuite déclinés par chacun dans le cadre de ses compétences disciplinaires; mais ces interactions à l'origine de leur construction garantissent que ces objets ont sens, à la fois, pour les chercheurs et leurs partenaires. Chacun des cadrans vise des productions spécifiques qui sont toutes utiles à des recherches ayant comme objectif une meilleure prise en compte des enjeux du développement durable dans les
${ }^{6} \mathrm{Qu}$ 'il distingue des modèles diffusionnistes et de la connaissance imparfaite qui ne sont pas très éloignés d'ailleurs de nos deux modèles précédents. 
ACTUALITÉS DE LA RECHERCHE problématiques environnementales ou agricoles s.I. II nous faut savoir reconnaître la pertinence de chacun, et ses limites, pour convenir des modalités de travail conduisant à des productions spécifiques et complémentaires.

La phase de négociation des termes de référence est cruciale dans de nombreuses situations : elle consiste en l'occurrence à traduire des problèmes concrets en questions de recherche traitables... car il est risqué de s'engager sur des objectifs qui ne pourraient pas être atteints, soit parce que les problèmes posés ne relèvent pas d'un travail de recherche, soit parce que l'équipe ne dispose pas des compétences requises, soit parce que le dispositif de recherche proposé n'est pas approprié. La dérive habituelle revient souvent à ne retenir que des questions de recherche traitables à partir des objets habituels des chercheurs : la démarche est ainsi inversée, le problème devient alors une situation d'application de concepts et de méthodes bien maîtrisées par les disciplines présentes. Les résultats sont certains, mais ils n'apportent le plus souvent pas d'éléments nouveaux susceptibles de permettre aux partenaires de modifier la situation qui leur pose problème. La créativité, si tant est qu'il y en ait encore, se cantonne du côté des chercheurs... Depuis plusieurs années, des approches alternatives sont expérimentées et mises en débat (Albaladejo et Casabianca, 1997). Une autre démarche consiste en effet à mobiliser des disciplines différentes, jugées nécessaires pour traiter les questions de recherche issues de la traduction des problèmes: $c^{\prime}$ est là une approche plus exigeante, car elle combine les difficultés de l'interdisciplinarité (différences de paradigmes, de concepts, de méthodes et de rythmes de travail entre disciplines) avec celles de la conduite du partenariat, justement du fait de ces différences de conception et de pratiques entre disciplines. C'est bien là tout l'enjeu de la mise en place d'un dispositif collectif de ce qui s'appelle dans la littérature, rechercheaction, recherche participative, recherche clinique, recherche intervention...

\section{Construire les objets socio-techniques en s'engageant dans l'action collective}

Dans le domaine des recherches participatives, on ne connaît pas a priori l'état des connaissances à la sortie, car celui-ci ne résultera pas exclusivement de la capacité explicative des expérimentations de la recherche, mais également des interactions entre les connaissances des chercheurs et les connaissances

${ }^{7}$ Ce que A. Hatchuel (2000b) qualifie d'émergence de nouvelles "figures d'acteurs ".

${ }^{8}$ Qui, eux, sont directement intéressés par la résolution des problèmes à l'origine des recherches et,

le plus souvent, dans un monde fait de concurrence et de compétitivité, pas seulement académique! la mettre en pratique, des objets identifiés pour l'instruire, des formes concrètes de collaboration pour la gérer ensemble, etc. Cette posture ne demande pas au chercheur moins de rigueur que les autres, puisqu'elle conduit à se mettre soi-même en cause et en situation d'apprentissage dans un dispositif complexe et hétérogène. Si l'on admet alors que l'universalité des connaissances scientifiques ne va pas de soi, il faut une grande exigence pour énoncer une production scientifique originale en termes théoriques et méthodologiques au delà de la situation spécifique dans laquelle on est amené à travailler : qu'est-ce qui est utile ici (pour les autres) et qu'est-ce qui est transportable ailleurs et donc justifiable et valorisable du point de vue de la recherche publique? On s'intéresse alors à la production de connaissances génériques et non plus seulement de connaissances à valeur générale. Les résultats de telles recherches portent ainsi autant sur les principes de raisonnement, la pertinence des modèles produits ou utilisés, les dispositifs conçus pour permettre le travail collectif, l'identification de phases différenciées dans le processus de collaboration, l'analyse des référentiels et des lieux à l'origine de l'élaboration des idées sous-tendant les actions, que dans des données originales produites in situ ou de nouvelles normes localement satisfaisantes. Ainsi, la relation opérationnelle avec les « acteurs " ne passe pas exclusivement via des outillages particuliers issus de modèles valides, mais bien souvent par une reconstruction de l'espace du problème et la conception de nouvelles formes d'action du fait des apprentissages croisés.

Il faut de la rigueur également pour analyser les transformations que ces apprentissages permettent, les différenciations sociales qui accompagnent ces processus et qui se matérialisent par l'apparition de nouveaux métiers, rôles, statuts, droits ${ }^{7} \ldots$ et partant les nouveaux rapports de domination et de coopération qui peuvent être ainsi induits : la recherche doit s'assurer des nouveaux pouvoirs qu'elle génère chez certains de ses partenaires ${ }^{8} \ldots$ surtout si $c^{\prime}$ est au détriment de certains de ses autres partenaires! Des connaissances nouvelles changent les situations qui sont à l'origine des problèmes et conduisent à l'identification de nouveaux objets sur lesquels les accords entre acteurs peuvent se réaliser ; c'est heureux, mais il serait bien souvent irresponsable de ne pas s'en préoccuper en tant que chercheurs. Comment les chercheurs pourraient-ils rester indifférents et passifs, $d$ 'un point de vue institutionnel ou professionnel, aux nouveaux objets de gouvernements qu'on voit émerger dans le domaine de l'environnement autour de questions comme la qualité des eaux, les risque d'inondation, les incendies de forêt, la gestion des zones humides, du traitement des déchets, de conservation ou de réhabilitation des paysages?

En effet, ces nouveaux accords se constituent autour d'objets socio-techniques que nous pouvons qualifier "d'objets intermédiaires ", au sens de Vinck (1999), dans la mesure où ils relient entre eux des entités hétérogènes et où ils marquent les étapes successives d'un projet collectif, à la suite de chaque épreuve que se sont donnés les acteurs pour le construire. Ces épreuves sont révélatrices des positions de ces acteurs, mais 
également des connaissances qui sont en jeu et qui s'élaborent en cours d'action. La connaissance est comprise ici comme le point de vue d'un acteur, construit dans l'action, sur un énoncé et les entités qu'il engage : elle désigne, ainsi que le rappellent Berger et Luckmann (1986), la relation qu'un acteur entretient avec ces entités. Dans une dynamique collective, les détenteurs de ces connaissances occupent des positions asymétriques et, sans développer ce point ici, soulignons au passage que celles des chercheurs sont particulièrement cruciales, car ils ne justifient leur présence que par les connaissances qu'ils produisent. Ils doivent donc accorder une vigilance particulière à expliciter le point de vue qu'ils ont choisi et les objets qu'ils étudient. Ainsi, c'est « l'action, ou mieux l'interaction dans laquelle ils sont engagés, qui [...] donne force, sens et réalité effective " aux objets et aux acteurs pris ensemble dans le processus même de l'interaction (Jeantet, 1998). C'est pourquoi, il est essentiel de qualifier ces objets et d'étudier scientifiquement les modalités de leur construction, leurs contenus et les rôles qui leur sont donnés dans l'action collective. Ces objets peuvent prendre la forme de contenus de cahiers des charges, de plans d'action, de la définition de « bonnes pratiques", de formes et de dates de pratique de telle ou telle activité (chasse, mise en eau, pâturage...) etc. Nous avons montré par ailleurs (Hubert et Bonnemaire, 2000) qu'il est possible d'en rendre compte à partir d'une démarche scientifique interdisciplinaire et que cela est, de plus, susceptible de renvoyer à des interrogations particulièrement fécondes du point de vue des différentes disciplines engagées. C'est essentiellement par l'implication de chercheurs dans le cadran « holistique " que ces objets peuvent être identifiés et qualifiés en regard du rôle qui leur est donné (et des éventuels changements de rôle au fil des processus d'interaction) et qu'ils sont alors susceptibles d'être déclinés - de façon à en étudier les diverses facettes - par d'autres disciplines selon leur propres paradigmes... c'est-à-dire dans le cadran de leur choix !

Il s'agit bien de s'engager alors dans ces processus incertains que sont les dynamiques d'action collective en s'assurant de la maîtrise de cet engagement par leur théorisation. On peut ainsi concevoir que le pilotage de tels processus collectifs pourrait s'identifier à l'accompagnement d'un nouveau procédé, selon une triple logique : d'acceptabilité (par les partenaires concernés, car cela ne se fait pas s'il n'y a pas accord sur la coopération), de projet (avec des objectifs, des étapes, des participants identifiés et engagés, car il s'agit bien de concevoir un futur, de construire un devenir) et de recherche (des explorations, des expérimentations, des prototypes, etc.).

L'espace de l'action collective est ainsi à considérer comme un espace de reconstruction simultanée des savoirs des acteurs et des savoirs des chercheurs, ainsi que des relations entre acteurs et entre acteurs et chercheurs, en distinguant, ainsi que le proposent Hatchuel et Weil (1999) : I'espace des concepts d'action qui sont au fondement du projet (c'est-à-dire qui désignent des objets, des produits, des tâches dans lesquels celui-ci s'incarne) et l'espace des connaissances qui permettent de mettre en œuvre concrètement ces concepts d'action. Pour ces auteurs, l'engagement du chercheur contribue alors essentiellement à un travail de "dénaturalisation/régénération » : en d'autres termes, il s'agit de s'interroger sur les normes, concepts, critères d'efficacité des actions engagées dans l'action collective, afin de les remettre en cause et de les transformer en fonction des changements en cours, dans un double mouvement, à la fois de révélation de faits connus et de faits non perçus, et de création de connaissances et de relations nouvelles (Couix et Hubert, à paraître). Ainsi, le processus de recherche n'est pas dissociable du processus de décision, l'investigation est pleinement reconnue comme constituante de la conception de solutions, conduisant à des choix explicites.

Afin de concevoir et mettre en œuvre le pilotage de ces processus, il s'agit de contribuer à la constitution de dispositifs d'action collective, prenant en compte I'hétérogénéité des acteurs concernés et la dimension historique des situations et des configurations institutionnelles. Plusieurs ouvrages illustrent de telles approches à partir de démarches empiriques et de cadres théoriques (Röling and Wagemakers, 1998 ; LEARN Group, 2000). Les concepts d'action et les connaissances, qui sont ainsi potentiellement produits, modifient l'état cognitif de l'ensemble des acteurs engagés, ce qui concourt alors à favoriser l'émergence de nouveaux métiers et rôles pour assurer ce pilotage. Ils sont également à l'origine d'apprentissages, non seulement cognitifs mais organisationnels, entre les acteurs impliqués dans un problème commun, débouchant sur des dispositifs inédits traitant à la fois de connaissances, de pratiques, de normes et de règles sociales et imaginant de nouveaux instruments d'incitation et de coordination (outils de médiation, séquençage des actions, évaluation in itinere, etc.) permettant un réel pilotage de tels processus innovants sur la durée. Ce sont bien là des objectifs et des objets de recherche qui devraient trouver toute leur place dans la programmation de démarches du type de celles qui sont à mettre en œuvre dans les "zones ateliers " du PEVS.

\section{RÉFÉRENCES}

Albaladejo, C., Casabianca, F., (Eds.), 1997. La recherche-action. Ambitions, pratiques, débats. Études et Recherches sur les Systèmes Agraires et le Développement, 30, Inra SAD, Paris.

Alphandéry, P., 1996. Les oiseaux, le parc et les agriculteurs de l'isthme du Cotentin. Études Rurales 141-142; 125-141.

Akrich, M., 1993. Les objets techniques et leurs utilisateurs. De la conception à l'action. In : Conein, B., Dodier, N., Thévenot, L. (Eds.), Les objets dans l'action. De la maison au laboratoire. Coll, - Raisons pratiques :, 4, EHESS, Paris, pp. 35.58.

Bawden, R.J., 1997. Learning to Persist: A Systemic View of Development. In: Stowell, F.A., Ison, R.L., Armson, R., Holloway, I., Jackson, S., McRobb, S. (Eds.), Systems for Sustainability. Plenum Press, New York and London, pp. 1.5 .

Berger, P., Luckmann, T., 1986. The social construction of reality. Doubleday \& Cy, New-York.

Billaud, I.P., 1996. Négociations autour d'une nature muette. Dispositifs environnementaux dans les marais de l'Ouest. Études Rurales 141-142. 63-83. 
ACTUALITÉS Callon, M., 1989. La science et ses réseaux. Genèse et circulation des faits scientifiques. La Découverte, Paris.

Callon, M., 1998. Des différentes formes de démocratie technique. Annales des Mines, 63-73.

Checkland, P., 1993. Systems Thinking, Systems Practice. J. Wiley \& Sons, Chichester, UK.

Couix N., Hubert, B., 2000. Promoting Collective Learning in a Land-Use Management Project: Thirteen years Experience in Researcher-Technician Partnership in the Cévennes, France. In : LEARN Group (Eds.), Cow up a Tree. Knowing and Learning for Change in Agriculture. Case studies from Industrialised Countries. Coll. «Science Update ", Inra Editions, Paris, pp. 121-140.

Couix, N., Hubert, B., (à paraitre). Mise en œuvre des politiques publiques d'aménagement de l'espace : une construction progressive du domaine de connaissances, Le Cas de la Défense de la Forêt contre les Incendies en Cévennes Alésiennes. Natures Sciences Sociétés.

Darré, J.P., 1997. Une condition de la Recherche-Action : la coopération sur la problématique et son évolution. In : Albaladejo, C., Casabianca, F. (Eds.), La recherche-Action. Ambitions, pratiques, débat. Étud. Rech. Syst. Agraires Dév. 30, 177-182.

Deverre, C., Mormont, M., Selman, P., 2000. Consensus building for sustainability in the wider countryside. Rapport final à I'Union européenne, contrat $n{ }^{\circ}$ ENV4-CT96-0293.

Dodier, N., 1993. Les appuis conventionnels de l'action. Éléments de pragmatique sociologique. Réseaux 62, 63-85.

Dodier, N., 1997. Remarques sur la conscience du collectif dans les réseaux sociotechniques. Sociologie du travail 2, 131-148.

Hatchuel, A., 2000a. Intervention Research and the Production of Knowledge. In : LEARN Group (Eds.), Cow up a Tree. Knowing and Learning for Change in Agriculture. Case studies from Industrialised Countries. Coll. Science Update, Inra Editions, Paris, pp. 55-68.

Hatchuel, A., 2000b. Prospective et gouvernance : quelle théorie de l'action collective? In : Heurgon E., Landrieu J. (Eds.), Prospective pour une gouvernance démocratique. Colloque de Cerisy. Édition de l'Aube, Paris.

Hatchuel, A., Weil, B., 1999. Design-oriented Organizations. Towards a unified theory of design activities, 6th International Product Development, Management conference, Cambridge (UK), July 5-6 1999, 28p.
Hubert, B., Bonnemaire, 1., 2000. La construction des objets dans la recherche interdisciplinaire finalisée : de nouvelles exigences pour l'évaluation. Natures, Sciences, Sociétés 8 (3), 5-19.

Hubert, B., Moulin, C.-H., Roche, B., Pluvinage, J., Deffontaines J.P. (à parâ.tre). Quels dispositifs pour conduire des recherches sur problèmes en partenariat ? L'intervention d'une équipe de recherche au Pays basque intérieur. Économie Rurale.

Jeantet, A., 1998. Les objets intermédiaires dans la conception, Éléments pour une sociologie des processus de conception. Sociologie du travail 3,291316.

LEARN Group, 2000. Cow up a Tree. Knowing and Learning for Change in Agriculture : case Studies from Industrialised Countries. Coll. Science Update, Inra Editions, Paris.

Legay, J.M., 1993. Questions sur la dynamique de l'exploitation halieutique. Préface. Actes de la table ronde Orstom/liremer Montpellier, septembre 1993. In : Laloé, F., Rey, H., Durand, I.L. (Eds.). Questions sur la dynamique de l'exploitation halieutique. Orstom éditions, Paris, pp. 9-11.

Legay, I.M., 1997. L'expérience et le modèle. Un discours sur la méthode. Coll. - Sciences en questions s, Inra édition, Paris.

Miller, A., 1983. The Influence of Personal Biases on Environmental ProblemSolving. J. Environmental Management 17, 133-142.

Miller, A., 1985. Technological Thinking: Its Impact on Environmental Management. Environmental Management 9 (3), 179-190

Mormont, M., 1996. Agriculture et environnement : pour une sociologie des dispositifs. Économie rurale 236, 28-36.

Pavé, A., 1994. Modélisation en biologie et en écologie. Éditions Aléas, Lyon.

Priêto, L., 1975. Pertinence et pratique. Essai de sémiologie. Éditions de Minuit, Paris.

Röling, N., Wagemakers, A. (Eds.), 1998. Facilitating Sustainable Agriculture. Participatory Learning and Adaptive Management in Time of Environmental Uncertainty. Cambridge University Press, Cambridge.

Roybin, D., Fleury, P., Béranger, C., Curtenaz, D., 2001. Conduite des recherches pluridisciplinaires en partenariat et apprentissages collectifs. Le cas du GIS Alpes du Nord. Natures Sciences Sociétés 9 (3), 16-28.

Vinck, D., 1999. Les objets intermédiaires dans les réseaux de coopération scientifique. Rev. franç. de sociologie XL-2, 385-414. 